\title{
PRINCIPIOS ORGANIZADORES EN SEIS MEDIOS PERIODÍSTICOS EMERGENTES EN INTERNET
}

\author{
ORGANIZING PRINCIPLES IN SIX DIGITAL JOURNALISM'S \\ EMERGING PLATFORMS
}

\author{
PRINCÍPIOS ORGANIZADORES EM SEIS MEIOS JORNALÍSTICOS \\ EMERGENTES NA INTERNET
}

Por:

Kevin Alexis García

Escuela de Comunicación Social

Universidad del Valle

Kevin.alexis.garcia@correounivalle.edu.co

Resumen: Este artículo presenta los resultados de una investigación realizada en el marco del Observatorio de Fenómenos Emergentes de Información de la Universidad del Valle. Busca dirimir el patrón en las prácticas de seis medios periodísticos latinoamericanos emergentes en la web para, a través de ellas, esbozar las claves por las cuales están pasando las transformaciones del oficio y las influencias de las tecnologías del computador y el internet. Conjunto de fenómenos que hoy transforma todo el campo informativo.

Palabras Clave: Medios Latinoamericanos, Fenómenos Emergentes, Transmedia, Teoría de Sistemas, Complejidad.

\begin{abstract}
: this article exposes the results of a research carried out in the context of the Emerging Phenomena of Information Observatory at Universidad del Valle. The text aims to show the pattern in the practices of six digital journalism's emerging platforms from Latin America in order to outline, through them, the key points by which transformations are taking place in journalism as a profession and the influence of technology and internet on it, which are a set of phenomena that transforms the whole informative field.
\end{abstract}

Keywords: Latin American Media, Emerging Phenomena, Transmedia, Systems Theory. 
Resumo: este artigo apresenta os resultados de uma pesquisa realizada no contexto do Observatório de Fenômenos Emergentes de Informação da Universidad del Valle. O texto visa a dirimir o padrão nas práticas de seis meios jornalísticos latino-americanos emergentes na web para, através delas, tracejar as chaves pelas quais passam as trasnformações da profissão e a influência da tecnologia do computador e da internet, os quais são um conjunto de fenômenos que hoje transformam o campo informativo.

Palavras-chave: Mídia Latino-americana, Fenômenos Emergentes, Transmídia, Teoria de sistemas.

Cuando Gutenberg inventó la imprenta en 1452 tenía una clara conciencia de las pretensiones de su nueva tecnología. Su invención agilizaría de una forma fantástica la producción de libros que hasta el momento eran realizados de forma artesanal y demandaban un alto esfuerzo de mano de obra. Cincuenta años después de creada, en el mundo se había producido un número de libros igual al que había producido toda la humanidad en los últimos mil años. El dato lo brinda Carr (2011) y significa toda una provocación para comprender el impacto de las tecnologías en la vida social.

Desde 1990 se ha iniciado un proceso de masificación global de las tecnologías del internet. Pasados 25 años, el impacto de su transformación es notable. La emergencia de estas tecnologías ha significado una amenaza para la industria del papel, y ha generado un remezón en la industria del periodismo impreso, una situación que nos lleva a analizar las posibilidades de que continúe siendo una industria sostenible o, por lo menos, de que se conserve tal como lo venía haciendo desde la segunda mitad del siglo XX, etapa en que se populariza la televisión llevándose consigo gran parte de la inversión publicitaria.

Las evidencias saltan a la vista y van configurando paulatinamente un panorama general de reacciones encadenadas que contraen la industria informativa: fuerte reducción de la venta de periódicos, reducción de lectores, reducción de la inversión publicitaria en prensa escrita, reducción de puestos de trabajo, insostenibilidad de algunos medios hasta su desaparición y puesta en venta de periódicos tradicionales por parte sus propietarios históricos. También una reconversión de históricos medios impresos en medios híbridos y o digitales.

Según los reportes de la Newspaper Association Of America ${ }^{1}$ el promedio de circulación diario de copias de periódicos en el año 2000 en Estados Unidos era de 55.773 ejemplares; para el 2011 esa cifra ya se había reducido a 44.421, la cantidad más baja de los últimos 60 años. Un retroceso cuya cifra solo es un poco superior al tiraje diario que tenía este país en 1940. 
Alemania, la nación más poderosa de la Unión Europea, ha experimentado un proceso similar. Para 1954 circulaban en Alemania cada día 225 periódicos, pero esta cifra se redujo para el 2014 a 129. En Francia el diario Le Monde pasó de vender en el 2001 un promedio de 405.000 periódicos, a vender en 2014, un promedio de 298.000 copias diarias.

El panorama en internet ofrece un movimiento contrario. En solo un año la población global con acceso a la red aumentó en doscientos millones; pasó de 2710 millones en 2013, a 2920 millones en el 2014. El mayor acceso a las computadoras, los procesos de modernización tecnológica que han venido desarrollando los países y la masificación de los teléfonos inteligentes han favorecido el acceso y uso del internet. A los entornos virtuales han migrado los lectores, y en dicho entorno las redes sociales alcanzan los mayores tiempos de uso activo. Facebook registró una actividad en 2014 de 1.300 millones de cuentas de usuarios. Esta condición ha atraído a la industria publicitaria que ahora encuentra en los medios virtuales una gama más amplia de clientes que pueden ver sus anuncios.

Muchos periódicos en el mundo han tomado la decisión de publicar sus contenidos en internet para reducir sus gastos y mantener lectores. Otros han apostado por un sistema mixto, un proceso de transición mediante el cual ofrecen sus contenidos en línea de forma gratuita o mediante suscripción pagada, en una búsqueda de monetización sostenible. Ese contexto empieza a ofrecer un escenario creciente para medios como The New York Times, que ha venido registrando un aumento de suscriptores a su portal digital y alcanzó 831.000 hacia finales del segundo trimestre de $2014^{2}$. En conjunto, en marzo de 2014 los periódicos registraron 161 millones de visitantes únicos en sus contenidos digitales. En octubre del mismo año la cifra se elevó a 166 millones ${ }^{3}$.

La nueva matriz de medios de comunicación se vislumbra entonces altamente virtual, signada por la influencia de múltiples plataformas y redes sociales. Allí los medios periodísticos podrían disponer un escenario estratégico para subsistir y el campo periodístico general un nuevo ecosistema para mutar y florecer.

Esta investigación es parte de un programa de investigaciones sobre la emergencia y transformaciones generadas por las tecnologías de la web. Se sincroniza, dialoga e incorpora conceptos y perspectivas críticas de autores como Landow (2009), Levy (1999) y Manovich (2013). Es la tentativa de un plano general que apunta su valor a intentar mirar el bosque. Para ello no procura tanto descripciones densas de objetos puntuales, pretende sobretodo ofrecer un sistema (Capra, 1998) de conexiones, conectar los puntos de una constelación, procurar una perspectiva de conjunto sin la pretensión de agotar un objeto que continúa emergiendo y transformándose. Este es un corte sincrónico. Un ejercicio de hermenéutica de los nuevos medios (Manovich, 2005) para el cual combino investigación teórica, análisis de objetos empíricos, rastreo de fenómenos emergentes e interpretación, tendientes hacia una investigación aplicada en el diseño de asignaturas para la enseñanza del periodismo en internet. 
¿Cuáles son las principales características de los nuevos medios?, ¿qué transformaciones han propiciado en el ejercicio de disciplinas como el periodismo?, ¿qué

transformaciones ha sufrido el concepto de noticia periodística?, ¿cómo se expresan los fenómenos de convergencia de medios y lenguajes?, ¿en medio del pesimismo cultural y el fetichismo tecnológico, qué análisis menos maniqueos pueden proponerse?, ¿cómo se transforman los roles del periodista en internet?, ¿cómo se transforman los criterios de noticiabilidad y la agenda informativa?, ¿qué nuevos oficios ligados al periodismo están surgiendo y qué técnicas y prácticas para la investigación?

El horizonte de interrogantes es amplio y en esta etapa pretendemos abordar algunos de los rasgos más expresivos. Para ello se ha diseñado una investigación exploratoria que mediante el Análisis cualitativo comparado de medios emergentes en internet investiga los patrones de un naciente sistema de medios que podrían brindar horizontes hacia la exploración del periodismo del futuro. Se busca dirimir aspectos de su sustancia (estructura) y de su forma (patrón, orden, cualidad del medio).

Desde una perspectiva sistémica y para el corpus puntual nos preguntamos ¿cuáles son las principales características de los fenómenos emergentes de periodismo en internet?, ¿se podría identificar un patrón en sus prácticas?, ¿cuáles son sus modos de autoorganizacion y autoreproducción?, ¿cuáles son los tejidos y conexiones que establecen? ¿Qué ecosistema los posibilita y qué características de los nuevos medios aprovechan? ¿Qué estrategias de sustentabilidad emplean? ¿De qué forma se adaptan a las variaciones del entorno (informático y lector)?

En medio de la crisis de la prensa escrita y de las mutaciones generadas por las tecnologías informáticas, han ido emergiendo medios independientes en internet. Para este estudio se han seleccionado medios que reúnen por los menos cuatro de los siguientes criterios: 1) Son medios nativos de internet, no migrados.

2) Fueron creados por periodistas (portadores de una historicidad del oficio). 3) Son independientes y no fueron concebidos como proyectos de negocio de corporaciones del capital. 4) Incorporan en sus procesos innovaciones que exploran el lenguaje de los nuevos medios. No son réplicas del periodismo industrial. A través de sus prácticas y contenidos se insinúan rasgos que podrían orientar prácticas y técnicas de vanguardia.Y 5) sus trabajos son reconocidos entre sus pares como muestra de buenas prácticas.

Cada medio seleccionado debió cumplir por lo menos cuatro de los cinco criterios para integrar el corpus. Esta medida buscaba seleccionar una muestra relativamente equitativa sin desconocer las particularidades de los contextos propios. Luego de un proceso de observación, rastreo y análisis empírico en internet, se configuró un corpus de siete medios que cumplían los criterios. El primero de ellos es $\mathrm{Ciper}^{4}$, un Centro de investigación periodística, creado en Chile en 2007. El segundo es Verdad Abierta, Conflicto armado en Colombia ${ }^{5}$, un medio fundado en 2008 en Colombia. 
El tercer medio, también colombiano, es la Silla Vacía ${ }^{6}$, creado en 2009. El cuarto es Chequeado $^{7}$, creado en 2010 en Argentina. El quinto medio es Connectas ${ }^{8}$, plataforma de periodismo para las Américas, fundado en 2012 desde Colombia. Finalmente, el sexto medio es Poderopedia ${ }^{9}$, fundado en 2012 en Chile.

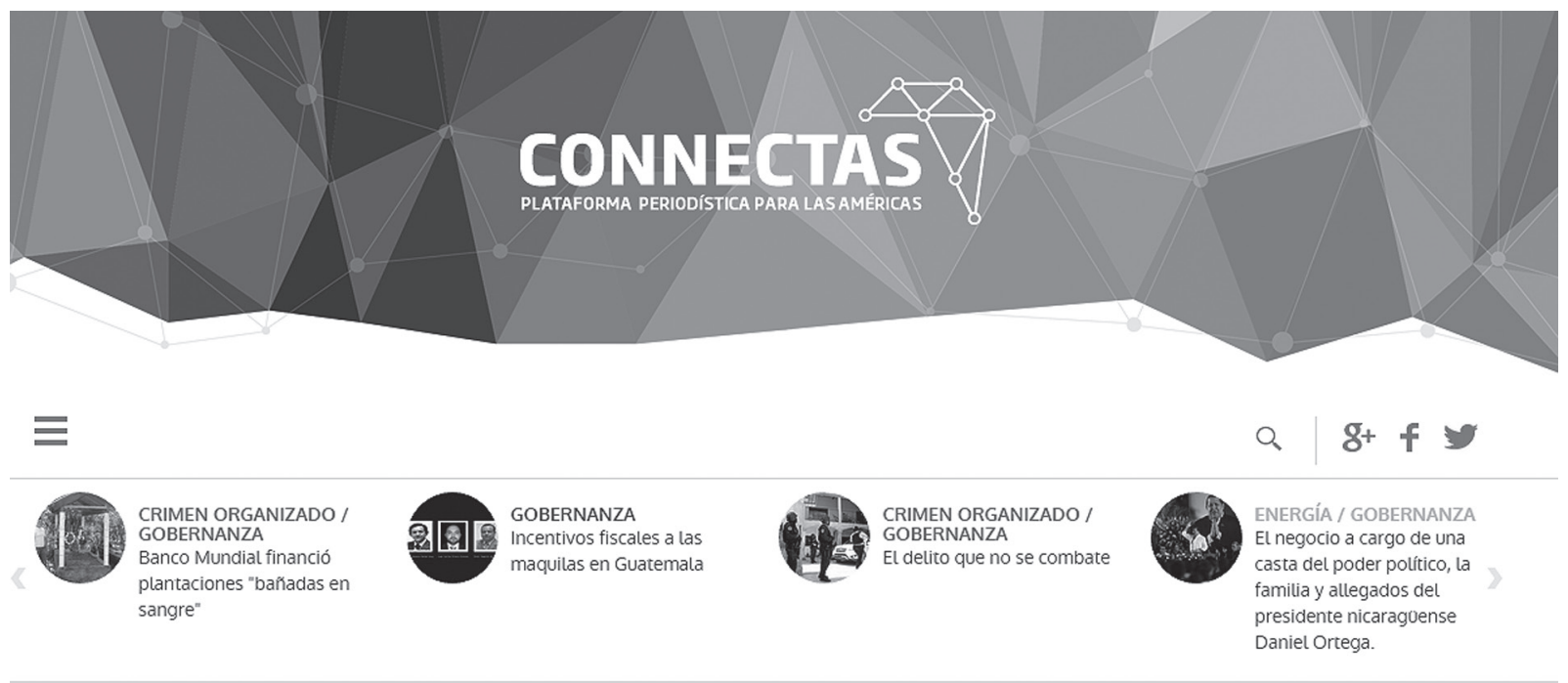

Imagen 1. Zona frontal superior de la página Connectas, Plataforma periodística de las Américas.

Para aproximarnos a la identificación de un posible patrón en sus prácticas se diseñó una matriz de registro con 30 categorías que permitieron clasificar datos sobre su configuración, estructura organizativa y operativa, así como la deontología de sus prácticas, modos de financiamiento y posibles modos en que creaban en otros países capítulos de sus proyectos, particularidades, exploraciones e innovaciones en sus contenidos y técnicas de trabajo.

El análisis comparado permitió identificar unas prácticas que permiten esbozar un patrón de conducta y autoorganización del sistema de medios independientes en internet que detallaré a continuación. Al igual que con los criterios de selección, cada característica no es cumplida en su totalidad por todos los medios, pero tiene un nivel de recurrencia entre la mayoría. Los hallazgos nos permiten deducir que los medios estudiados:

1. Son medios especializados, bien sea en un aérea temática, en un procedimiento del periodismo o en un tipo especial de periodismo. Los medios emergentes no pretenden ser misceláneos y han construido un enfoque de valor diferencial frente a los medios migrados, especialmente frente a los grandes medios industriales que arribaron al entorno informático. Ante ellos optan por un valor cualitativo antes que cuantitativo. La Silla Vacía es un medio especializado en la política colombiana y dicho énfasis está sugerido en su título. En Colombia varios hechos políticos han tenido como escenario y figuración una silla vacía: en la apertura del proceso 
de paz entre el gobierno del presidente Pastrana y las guerrillas de las Farc, la silla de Manuel Marulanda Vélez quedó vacía. En la movilización de las comunidades indígenas del norte del Cauca hacia Cali, el presidente Uribe anunció que se reuniría con ellos a su llegada a la capital y su silla quedó vacía. Durante el proceso de indagación de la parapolítica, se decretó la pérdida de investidura para los congresistas vinculados con paramilitares y se estipuló que sus sillas quedaran vacías en el Congreso. De estas situaciones este medio se ha valido para resignificar el símbolo de la silla en la política e invitar a los ciudadanos a ocuparla.

Por área también se ha especializado Verdad Abierta. Este medio fue creado en asociación entre la Fundación Ideas para la Paz y la Revista Semana. Fue pensado como una plataforma para ampliar junto con periodistas, investigadores, activistas sociales y comunidades, el conocimiento del fenómeno paramilitar en sus diversas dimensiones: la historia, los protagonistas, los cómplices, las víctimas, su lógica económica y política. Posteriormente, amplió su perspectiva hacia la investigación del conflicto armado en Colombia.

Por su parte, por procedimientos específicos (chequeo y mapeo) se han especializado los medios Chequeado y Poderopedia. Chequeado es un medio argentino autodeclarado como no partidista que se dedica a la verificación del discurso público y la promoción del acceso a la información y la apertura de datos. El medio se especializa en el chequeado de datos. Verifica las declaraciones de políticos, economistas, empresarios, personas públicas, medios de comunicación y otras instituciones formadoras de opinión. Analiza la consistencia de los datos y los hechos e informa sobre su veracidad, falsedad o posible incitación al engaño. Por su parte, el medio chileno Poderopedia se ha especializado en el mapeo de las redes del poder en los negocios y en la política; ayuda a entender las relaciones entre las personas, las empresas y organizaciones de interés público para reflejar posibles conflictos de intereses y favorecer la transparencia.

El medio Connectas se ha especializado en el periodismo de carácter transnacional, en temas como infraestructura, energía, telecomunicaciones, tecnología, medio ambiente y crimen organizado. A Connectas no la define su área temática sino su marcado énfasis en un periodismo trasnacional y cooperativo entre las diversas naciones de las Américas. El Centro de Investigación Periodística Ciper, tal como lo indica su nombre, se ha enfocado en el periodismo de investigación. Procura una agenda propia que devela temas de los sectores públicos y privados en Chile. 
2. Están liderados por periodistas nodales. Con altas conexiones, personajes con trayectoria y relación con altas fuentes de información que gracias a su credibilidad pueden aglutinar recursos multiinstitucionales y recurso humano calificado. Esta es una característica recurrente. La Silla Vacía fue creada por Juanita León, una periodista de investigación con experiencia en los medios impresos El Tiempo y la revista Semana, los de mayor impacto en Colombia. Connectas fue creado por Carlos Eduardo Huertas, editor durante más de una década de la Unidad Investigativa de la revista Semana y en la actualidad Miembro del Comité Directivo de la Red Global de Periodistas de Investigación (GIJN). Integró el equipo ganador del premio Rey de España en 2008, y de los equipos ganadores del Instituto de Prensa y Sociedad Ipys Tilac, a la mejor investigación periodística en América Latina en 2007 y 2009. Ciper fue creado por Mónica González, una periodista con amplio reconocimiento en Chile que en 2010 recibió el Premio Mundial Unesco-Guillermo Cano de la Libertad de Prensa. Ha publicado más de cuatro libros. Fue editora de investigación del Diario La Nación. Similar característica de sus líderes también se cumple en los medios Verdad Abierta, Poderopedia y Chequeado. En estos medios emergentes No es el capital económico el mayor decisor, es el capital social y cultural. La legitimidad y la credibilidad sobre estos emprendimientos se conquistan a través de estas trayectorias. Estos periodistas disponen mapas de fuentes, conocen las estructuras institucionales, tienen temas e investigaciones previas y conocen las lógicas de funcionamiento de los medios, sus aciertos y desaciertos. Conocimientos que se constituyen en saberes valiosos que favorecen la sostenibilidad de los medios emergentes.

3. Sus integrantes constituyen salas de redacción pequeñas que pueden ensamblar sus trabajos (estilización del modelo posfordista). Son equipos de trabajo, metáforas de hormigas que producen en conjunto, ya no son los ermitaños de las unidades investigativas de los medios clásicos. Lejos están estas salas de tener equipos de cincuenta o más números de personas como solía ocurrir en los medios industriales. Estos medios emergentes operan con equipos que bordean las 10 personas en la mayoría de los casos, incluyendo entre ellos profesionales administrativos, diseñadores gráficos y programadores. Sin embargo, sus publicaciones evidencian un alto nivel y calidad. Se apoyan en equipos de trabajo externos a la sala de la redacción y ensamblan sus contenidos. Según Juanita León, directora La Silla Vacía, en su medio laboran alrededor de 12 personas pero en la producción de contenidos participan alrededor de 70 colaboradores. Por su parte Poderopedia, en Santiago de Chile, su ciudad de origen, dispone un equipo de cinco integrantes, acompañado por un consejo asesor de 15 personas, conformado principalmente por periodistas y abogados. En Ciper el equipo tampoco supera las 10 personas. Estos medios estilizan la producción postfordista (González, 2004), mediante una producción descentralizada, en la cual los lectores tienen una alta participación: proponen temas, comentan las notas, realizan chequeos y aportan documentos. 
4. Sus periodistas se sustraen del ánimo de lucro corporativo pero tienen más participación con el área económica del proyecto, en procura de su sostenibilidad. Están informados de las fuentes de financiación, sus modos y convenios ${ }^{10}$. Esta característica tiene una relación directa con el punto anterior. Estos medios carecen de complejos departamentos de mercadeo y ventas; por tal razón son los propios directores, editores y en algunos casos periodistas quienes elaboran proyectos y gestionan recursos. Tienen un mayor sentido de pertenencia por su medio, lo entienden como una obra en proceso. Favorecen la construcción de una identidad profesional mediante el desarrollo colectivo de un emprendimiento común. Los integrantes suelen participar de actividades de intercambio de saberes con otros periodistas.

\section{Se sienten en una etapa de transición hacia la} sustentabilidad y para lograrlo diversifican sus servicios evitando comprometer su independencia.

Estos medios están más cercanos a actividades de educación que de mercadeo. Sus periodistas realizan actividades de capacitación contratadas gracias al prestigiamiento que van adquiriendo los medios emergentes que se suman a las trayectorias individuales. Verdad Abierta ha participado de proyectos con la Oficina del Alto Comisionado para la Paz, en Colombia, en talleres de actualización y construcción de agenda con periodistas de medios locales. En capacitaciones cerradas informan sobre el estado del conflicto en las regiones, los actores protagónicos y propician la construcción de temas de investigación. Los integrantes de La Silla Vacía dictan talleres de alfabetización digital, charlas y seminarios, consultorías y participan de actividades de cooperación en proyectos internacionales. Chequeado es uno de los proyectos que ha desarrollado un gran de número actividades inherentes al chequeo de datos. Chequeos colectivos con desarrolladores, periodistas, programadores y diseñadores para el desarrollo de plataformas de chequeo electoral; así como la creación de Jusiticiapedia, un mapa de relaciones entre operadores del Derecho: jueces, fiscales y abogados; ha realizado asistencia técnica y acompañamiento a medios de Centroamérica; desarrollo de una aplicación móvil de chequeo, también realizan actividades transmedia de chequeo con estudiantes de secundaria; desarrollan una plataforma de e-learning para dictar capacitaciones de verificación de datos de forma remota; talleres de periodismo de datos para medios provinciales argentinos, seminarios teórico prácticos y desarrollan materias de periodismo de datos para programas de posgrado.

\section{Procuran resituar los pactos de independencia y} transparencia con los lectores. Vinculados a la esfera pública, los medios de comunicación tradicionales son empresas informativas, atienden lógicas empresariales y producen contenidos regulados por sus expectativas de lucro. En ocasiones los medios tradicionales hacen parte de una dinámica comercial, de un conglomerado económico con inversiones e intereses en otros sectores de la sociedad. Esta situación afecta la credibilidad con sus audiencias. En los encuentros de periodismo suelen escucharse relatos acerca de censuras y sobre todo autocensuras por parte de los periodistas en la elección de sus temas para evitar discordias con sus empleadores. Además de estas prácticas, los cambios de enfoque, la invisibilización de hechos noticiables para evitar afectación de intereses corporativos, la disminución de la intensidad en el cubrimiento de un hecho y el ocultamiento de información son procedimientos que se constituyen como sesgos a la hora de dar cuenta de la realidad. Frente a ese contexto, los medios emergentes independientes representan un espacio de mayor libertad de expresión para los periodistas. Sus integrantes hacen explícito el contrato implícito que establece el periodismo con la ciudadanía, se fijan esquemas de transparencia, publican sus donantes y anuncian sus fuentes de ingreso. Asumen el proceso de consolidación como una experiencia y acordes con la sensibilidad de la época dejan ver el proceso de construcción del medio. 
7. Son medios y metamedios que también pueden operar como transmedios. La mayoría de los medios se autodefinen subsidiarios de otros medios, sus periodistas entienden el medio como una fuente abierta a la ciudadanía pero también como un metamedio que provee contenidos y apoyo a otros medios. Verdad Abierta busca servir de fuente abierta a los demás medios de comunicación brindando información de profundidad sobre el conflicto armado. La Silla Vacía, busca aportar perspectivas críticas a los generadores de opinión, muchos de los cuales son columnistas y expertos consultados y divulgados en los grandes medios. El Centro de Investigación Periodística Ciper realiza convenios con medios impresos en Chile para proveer contenidos y divulgar sus investigaciones. Actividad similar es realizada por Chequeado que mediante convenio contractual publica sus chequeos en algunos medios nacionales argentinos. La plataforma Connectas tiene una fuerte perspectiva colaborativa entre medios del continente y favorece principalmente el establecimiento de redes de cooperación y cocreación. Poderopedia mapea las redes de poder y ofrece visualizaciones de datos que por su nivel de especialidad se constituyen sobretodo en fuentes de consulta de medios informativos. Esta situación podría entenderse como una estrategia para lograr, principalmente, una mayor visibilidad y trascendencia de sus trabajos. En medio de las mutaciones del entorno tecnológico y la emergencia de redes sociales, estos medios pueden operar como transmedios, es decir, pueden operar de forma descentralizada, construir perspectivas críticas y analíticas según sus áreas y procedimientos intereses en plataformas como Twitter, Facebook, Youtube, también en aplicaciones consultadas por smartphones sin que los usuarios necesariamente accedan al hosting del medio.

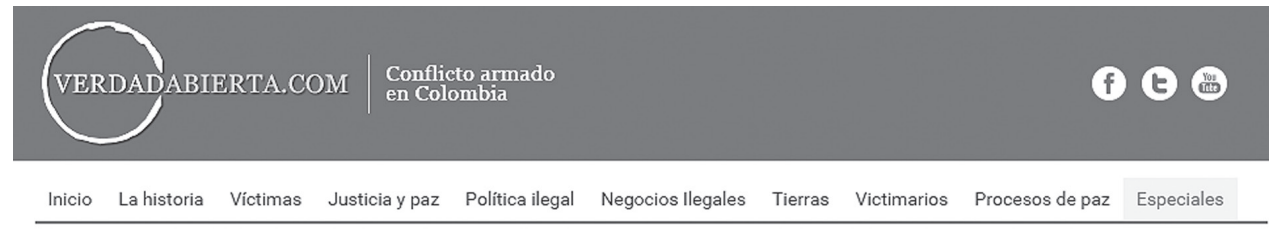

\section{Especiales}

Publicado el Jueves, 15 Enero 2015

FIMegusta 3 Twittear $\begin{cases}0 & 8+1 \\ 0\end{cases}$

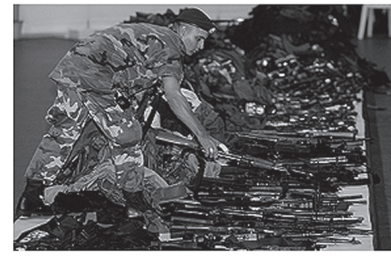

DOSSIER DESMOVILIZADOS

El largo camino para volver a la vida civil

Entrega de armas del Bloque Cacique Nutibara en la capital antioqueña en noviembre de 2003. Foto: archivo

Semana. Desde 1990 Colombia ha

reincorporado a la vida civil a miles de.

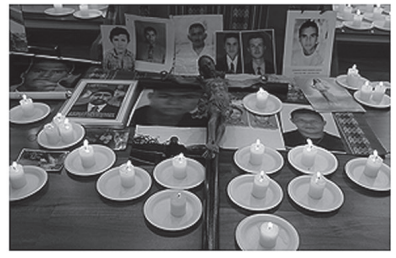

ASESINATOS SELECTIVOS Falsos positivos: una herida que sigue abierta

La noticia de que miembros del Ejército habian cometido miles de ejecuciones extrajudiciales estremeció al país hace siete años. Sin embargo, pasado el escándalo, las investigaciones tienen pocos avances.

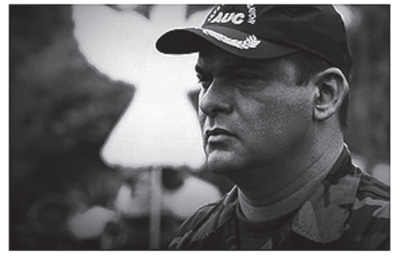

ESPECIAL MANCUSO DOSSIER

El dossier de Salvatore Mancuso Salvatore Mancuso, jefe de cuatro bloques de las Autodefensas Unidas de Colombia (Auc) y autor de miles de crímenes fue sentenciado por los tribunales de Justicia y Paz a..

Imagen 2. Sección de contenidos Especiales del portal Verdad Abierta, Conflicto Armado en Colombia. 
8. Tienen un carácter experimental y exploratorio entre sus prácticas. Acorde con las características del entorno informático, reconocen que están en un ambiente en constante transformación y exploración. Cada medio es una especie de obra en proceso de maduración y consolidación. Sin comprometer sus principios periodísticos, realizan con frecuencia ajustes, modificaciones y exploraciones en múltiples ámbitos. Como ha señalado Manovich la modularidad, la automatización y la variabilidad son algunas de las principales características de internet y los medios pueden realizar ajustes y modificaciones sin asumir grandes costos; el factor de riesgo y el costo de experimentación han disminuido de forma significativa en el entorno informático. Así, el equipo de La Silla Vacía durante buena parte de su proceso, se ha propuesto innovar un formato periodístico cada mes para contar historias. Estos formatos incluyen desde visualizaciones y mapeo de datos, bases de datos, fichas biográficas, actividades de verificación de información en tiempo real y curaduría de contenidos.

Poderopedia por su parte, a un lado de su logo indica en clave de superíndice su condición Beta (Poderopedia ${ }^{\text {Beta }}$ ), un concepto empleado en ingeniería de software para señalar una fase de desarrollo que ya reúne las características del conjunto del software pero que se encuentra en una etapa activa de eliminación de errores, de edición constante de ajustes. Los usuarios de un contenido clasificado como Beta, entienden que sus desarrolladores aún se encuentran mejorando el sistema, depurando sus procedimientos; se reconoce así que el medio es útil pero sigue siendo objeto de exploración y prueba.

El medio Chequeado explora constantemente la creación de nuevos desarrollos. Una de sus secciones más estables, la Zona de intercambio, le asigna a las revisiones de los usuarios la condición de "Chequeado en proceso", una condición exploratoria del dato como característica permanente. Connectas tiene una sección de Laboratorio, llamada Lab, nombre que vincula con los laboratorios de creación que a lo largo del mundo se están creando para conectar prácticas de áreas como el diseño, el arte y la ingeniería. En esta sección realiza constantemente foros de discusión acerca del periodismo para el nuevo milenio, las técnicas emergentes de investigación y en asociación con la Fundación para el Nuevo Periodismo Iberoamericano realiza los llamados Webinar, seminarios virtuales.

9. Participan de actividades convergentes y pluridisciplinares. Están relacionados con laboratorios de creación y experimentación, con hackers, desarrolladores, centros de entrenamiento $y$ de financiamiento. Asisten a un proceso de mutación cultural, de transformación de múltiples esferas de la sociedad, han creado sus medios en un proceso de transición y cambio de escala global y procuran prever las transformaciones del entorno. Chicas Poderosas $^{11}$, es un movimiento iniciado por Mariana Santos, becaria del ICFJ Knight, creado para capacitar periodistas en el uso de herramientas tecnológicas para la visualización de periodismo de datos en las salas de redacción. Sus jornadas combinan experiencias y consejos de expertos a través de charlas y talleres prácticos. 
Otra de las actividades que congregan periodistas e investigadores de medios son los Hackathon. Esta palabra sintetiza las palabras maratón y hackers. Desarrolladores, programadores, diseñadores y periodistas se reúnen para la creación de aplicaciones y plataformas de forma colaborativa en tiempos cortos. Ejemplo de productos creados en estas hackathon son las aplicaciones Hancel y Yointervengo. Ambas están en periodo de desarrollo, la primera favorece la comunicación segura mediante mensajes encriptados y la creación de un sistema de alarmas y monitoreo entre periodistas mediante ubicación satelital georeferenciada. La segunda aplicación permitirá que los usuarios establezcan conexiones entre contenidos off line y on line. La aplicación extraerá datos del Portal Único de Contratación, sistema en el cual se registran los procesos de contratación de las entidades públicas de Colombia, para que los ciudadanos puedan verificar la realización de las obras y su adecuada ejecución. De esta forma la aplicación permitirá construir una hoja de vida del contrato donde se establezcan relaciones entre el papel y la realidad.

Desarrollo de aplicaciones e interfaces, emergencia de nuevas sensibilidades de interacción, nuevas conceptualizaciones en la triada programación - mediosinteracción, nuevos usos y modos de acceso configuran coordenadas cruciales que los medios emergentes alternativos atienden con detalle.

10. No dan prevalencia a la jerarquía sino a la red. Tejen redes de intercambio, buscan expandir e interconectar sus propuestas con otros medios y organizaciones que comparten propósitos similares. Este es un elemento común entre la mayoría de la muestra analizada. Comprenden las ventajas de constituirse como sistema, de replicar sus prácticas y fidelizar audiencias. A diferencia de los medios tradicionales no promueven un discurso de propaganda en nivel jerárquico, es decir, ningún medio se autodefine mejor que los demás, buscan por el contrario replicar sus prácticas, capacitar, establecer acuerdos e interconectarse para ganar reconocimiento y apoyo. Por ello capacitan a profesionales de otros medios mediante eventos de asociaciones de periodistas y organizaciones de la sociedad civil. Varios medios se definen como plataformas colaborativas. Poderopedia tiene un apartado especial que instruye a los usuarios acerca de cómo crear un capítulo del medio en su respectivo país. Hasta el momento ha replicado su experiencia en Venezuela y Colombia, naciones en donde ya rastrean y visualizan las redes del poder político y económico. El medio Chequeado ha llevado su método de verificación a Colombia, ayudando a constituir la sección Detector de mentiras de La Silla Vacía. Así mismo, ha ayudado a replicar sus prácticas en Brasil, con la sección Truco de Agencia Pública y Preto no Branco de O Globo; en Uruguay con UYCheck y Verdadero o Falso de El Observador (solo en versión impresa); también ha replicado su experiencia en El Salvador con el Politígrafo de El Faro, y México con El Sabueso Verificador del portal periodístico Animal Político. Saben que en internet la elección de un medio no significa la exclusión de otros, a diferencias de los consumos físicos que implicaban la compra y la suscripción de los ciudadanos. 
11. Construyen agenda propia. Mediados por el ánimo de lucro y la libre competencia, los medios tradicionales suelen desarrollar fórmulas de confección de textos y criterios de noticiabilidad sobre la realidad que estereotipan y reducen la realidad misma. Las fórmulas que demuestran eficacia en términos de persuasión, impacto y lecturabilidad pocas veces sufren transformaciones significativas. Los medios tradicionales observan sus competencias y se autoreferencian en sus prácticas y contenidos. En otras palabras, los medios cubren lo que cubren los medios. Frente a este panorama, los medios emergentes alternativos procuran la construcción de agendas propias. Alejados de la noticia, elaboran trabajos reposados. Connectas se enfoca principalmente en proyectos especiales de largo aliento en áreas como el crimen transnacional, Poderopedia mapea redes del poder y en esa labor los datos no siempre tienen conexión directa con las discusiones nacionales del momento, Verdad Abierta tiene una agenda temática sobre el conflicto transversal a las coyunturas del día a día; Ciper tiene una agenda propia, apuesta por contenidos de alto impacto con ángulos distintos. Ejemplo de ello es su reportaje Viaje al fondo de la biblioteca de Pinochet, una indagación en los más de 55.000 libros que según el medio el exdictador atesoró a punta de regateos y fondos fiscales. Este trabajo obtuvo el Premio de la Fundación Nuevo Periodismo Iberoamericano y los jurados destacaron de él su capacidad para señalar nuevos aspectos de un personaje de quien se creía que los medios ya lo habían dicho todo. Ciper también se ha ocupado de realizar investigaciones sobre los desvíos en las utilidades de las instituciones universitarias definidas como entidades sin ánimos de lucro.

Frente a este conjunto La Silla Vacía procura comunicar desde el centro, situarse en la discusión nacional pero desde una perspectiva analítica e interpretativa, suministrando análisis de datos que permitan trascender las declaracioncitis de los medios tradicionales. A la par con este proceso construye trabajos de largo aliento que no son de coyuntura.

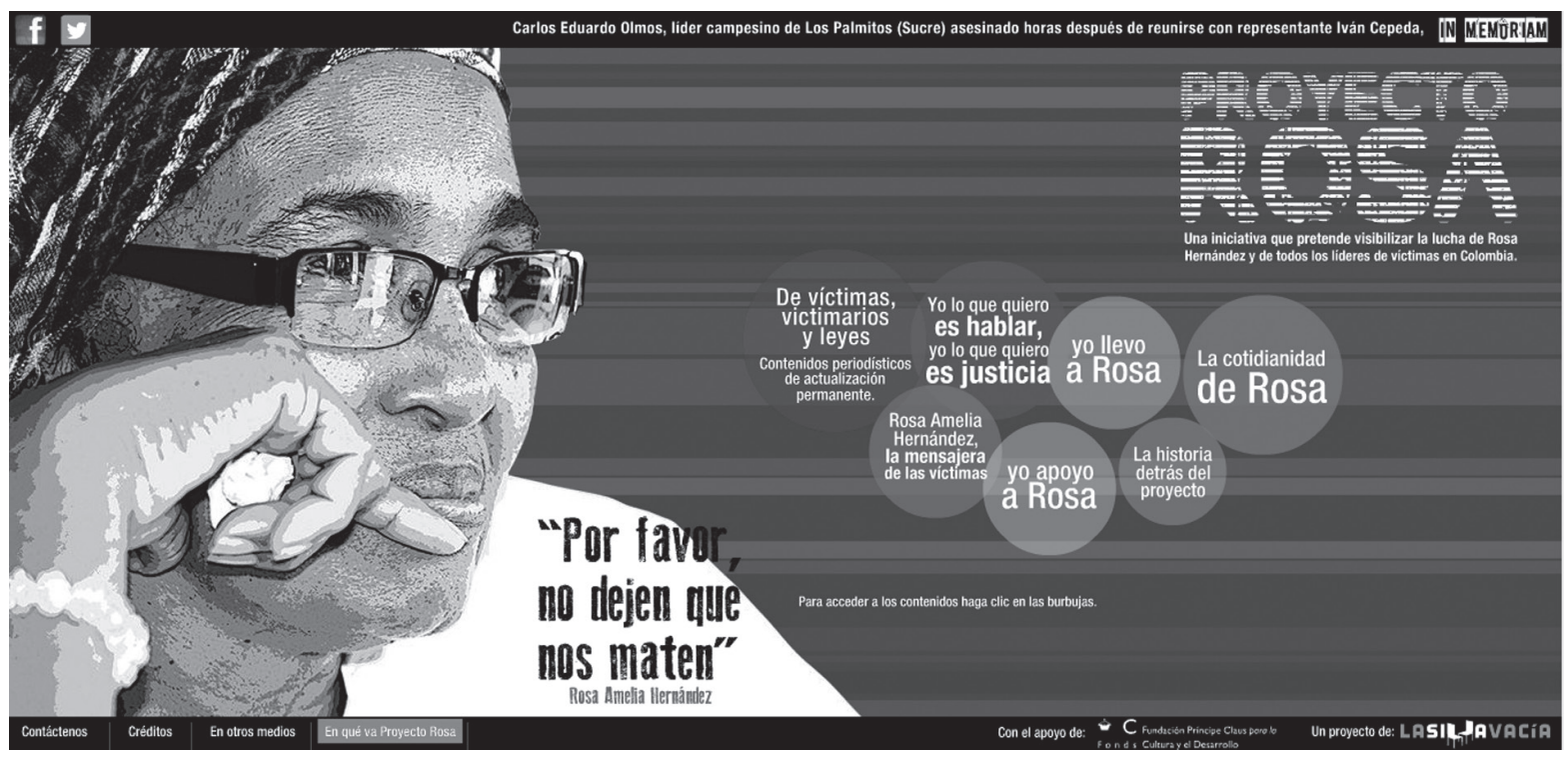

Imagen 3. micrositio Proyecto Rosa, creado por el portal La silla vacía, con recursos de donaciones de los usuarios. http://www.proyectorosa.com 
12. Se definen principalmente como medios abiertos y ofrecen a los usuarios los documentos que soportan sus investigaciones. Esta característica muy propia de la condición posmoderna pretende la construcción de un espacio de dominio público más amplio y el aumento de los bienes comunes de la información. Respecto al tipo de uso que los usuarios pueden hacer de sus contenidos, estos medios emergentes oscilan entre la reservación de sus derechos y la autorización para compartir, copiar y redistribuir el material en cualquier medio o formato con una finalidad no comercial, reconociendo la autoría y el tipo de intervención efectuada. Esta modalidad la permiten los medios Connectas, Poderopedia y Verdad abierta. Por su parte, los medios que reservan sus derechos son Chequeado, La Silla Vacía y Ciper. Entienden esta reserva como una estrategia en el camino hacia la sustentabilidad, ya que buscan proveer investigaciones a los medios tradicionales para obtener retornos por la inversión realizada durante la producción, así como visibilizar el trabajo del medio y ganar reconocimiento entre la ciudadanía. No obstante la diferencia de modalidades, todos estos medios operan como medios abiertos y divulgan bases de datos para que los ciudadanos realicen directamente sus propias consultas sobre los contenidos. Esta característica aún no se encuentra en medios tradicionales como los colombianos que migraron a internet.

\section{Promueven un periodismo activo, entienden la audiencia desde una perspectiva dinámica. Están interesados en fidelizar audiencias, interpelarlas, cooperar con ellas y darles un papel activo en la realización del medio. Para Juanita León sus lectores operan como especie de editores: suministran datos, revisan errores de redacción, verifican fuentes. Medios como Ciper, La Silla Vacía, Poderopedia y Verdad Abierta crean bases de datos para ser consultadas por usuarios; éstos deben formular sus propias preguntas, construir sus propias consultas activando filtros y criterios de extracción de los datos; los usuarios así abordan los contenidos a partir de sus propios interrogantes.}

Chequeado dispone de un módulo que permanece fijo a la vista del lector y que no altera su visibilidad a pesar de los clicks entre páginas y la navegación descendente de los lectores; siempre permanece estable. Se llama Zona de intercambio y a través de ella los usuarios pueden acceder a una red de verificación llamada Chequeador. Opera como una herramienta participativa ${ }^{12}$ mediante la cual los usuarios pueden realizar chequeos participativos. Cada usuario registrado puede sumar información a un chequeo que esté activo, también puede iniciar otro chequeo. Las participaciones y la información aportada por cada usuario podrán ser verificadas por los demás usuarios y calificada. El equipo de Chequeado se encarga de explicar la metodología y el orden. Todos los contenidos de esta sección permanecen siempre bajo la categoría de "chequeos en proceso". Esta sección es una zona de entrenamiento para los lectores en la verificación del discurso público. Los usuarios también pueden solicitar la realización de un chequeo al equipo de trabajo y subir sus propios datos. 
Uno de los fenómenos más llamativos del activismo político en internet en Colombia se vivió en las semanas previas a las elecciones presidenciales de 2010. En la primera vuelta de la votación, realizada el 30 de mayo, luego de resultado que daba ganador a Santos, emergió un grupo en Facebook llamado “¿Cuál primera vuelta?” al que en pocas horas se sumaron más de cuarenta mil usuarios, muchos de los cuales se dedicaron a inspeccionar los tarjetones de la Registraduría. Muchos de los simpatizantes de la Antanas Mockus encontraron que los votos consignados en el acta de los jurados (el formulario E-14) no coincidía con los reportados en el preconteo y casi siempre perjudicaban al candidato de La ola verde. Esta situación fue captada por la Silla Vacía que de inmediato convocó el apoyo de sus lectores y con más de sesenta de ellos, revisaron de manera aleatoria 1.010 mesas de votación ${ }^{13}$, que representaba cerca del $1.5 \%$ del total. Luego de la revisión y el cruce de datos, el equipo periodístico pudo concluir que se cometieron algunos errores el día de las elecciones por parte de los voceadores, pero que dichos errores no alteraban de manera significativa el resultado. Esta experiencia sentó un precedente acerca de las posibilidades de los medios para aglutinar y trabajar en equipo con sus audiencias. Convocar esfuerzos de un equipo de sesenta personas y realizar un conteo detallados de tarjetas de 1.010 mesas de votación demandaría una inversión de recursos bastante alta a un medio tradicional.

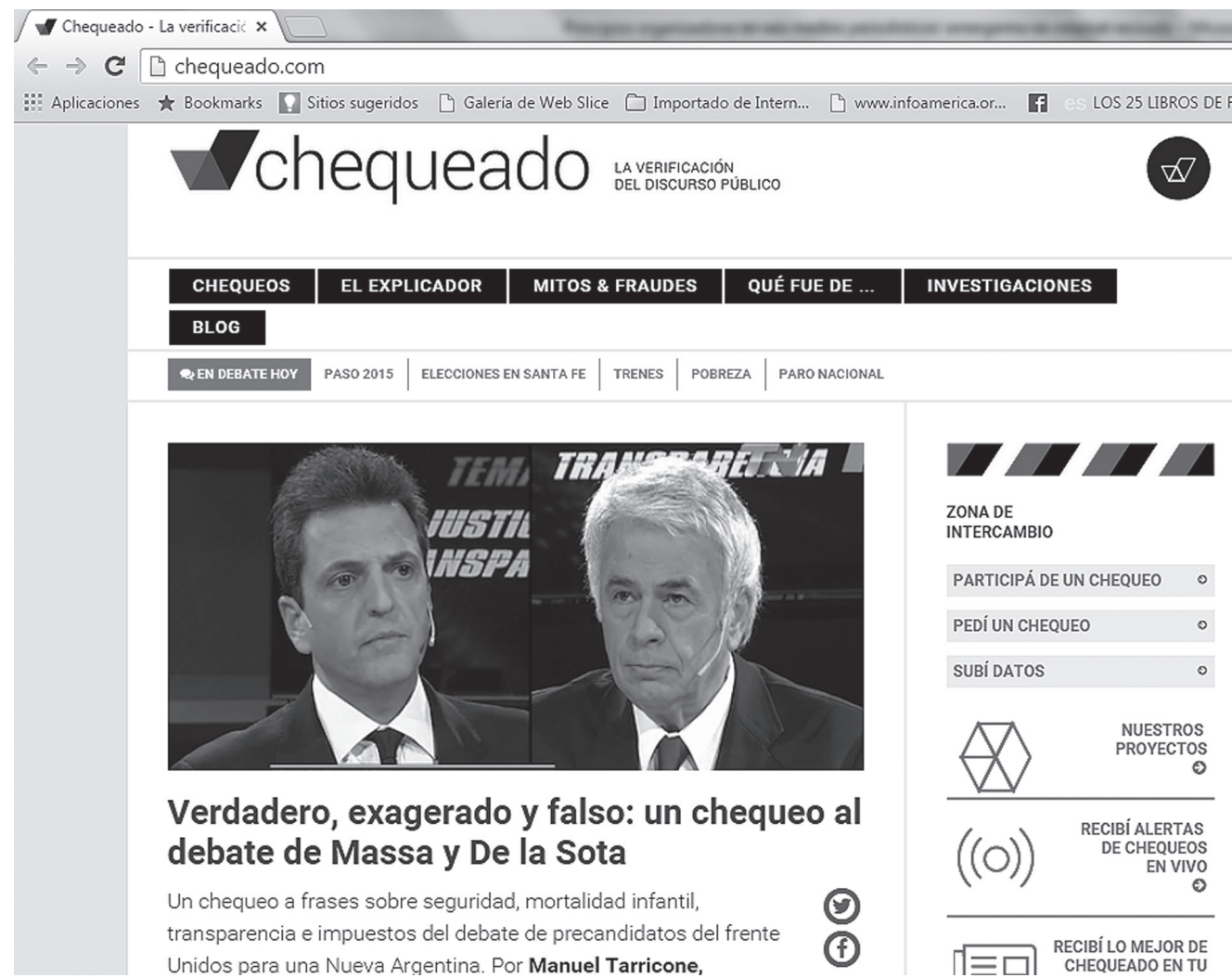

Imagen 4. Zona superior de la página principal del medio Chequeado, la verificación del discurso público. http: / / chequeado.com 
14. No recurren a la noticia como género dominante. A diferencia del periodismo informativo clásico, en estos medios emergentes predomina una convergencia de géneros y formatos: el fact checker, la ficha biográfica, la historia (Story) y el reportaje de profundidad. El nuevo ecosistema informático ha favorecido una ruptura de la mediación entre las fuentes y las audiencias. En internet, los medios ya no dan la noticia. Los ciudadanos pueden establecer vínculos directos con sus fuentes de interés a través de las redes sociales. Instituciones del Estado, políticos, líderes de organizaciones de la sociedad civil, activistas, deportistas y demás agentes de interés público hoy expresan directamente sus posiciones a través de Twitter, Facebook, Instagram, y a través de sus propios medios como portales, páginas y blogs. Frente a ese escenario, los medios seleccionados buscan establecer como valores diferenciales la conversación informada, la interpretación, el análisis y la curaduría de contenidos. Ninguno de estos medios se considera noticioso y ninguno usa la noticia como género dominante.

Chequeado analiza los datos de interés que circulan en el ámbito público; su texto dominante podría llamarse un fact checker, un chequeado de datos. Este texto contextualiza el origen del dato, presenta las fuentes originales, oficiales y alternativas que lo interpretan, confirma, relativiza o desmiente la información y califica el dato. Por su parte La Silla Vacía explora formatos de expresión que implican desarrollo de código de programación y visualizaciones de datos. La dimensión discursiva textual se soporta sobre historias, entendidas en analogía con la tradición anglosajana de story (León, 2011). Ciper recurre al reportaje de investigación y de profundidad, piezas de largo aliento que combinan información, interpretación y análisis. Una perspectiva semejante a Ciper es asumida por Connectas, medio que apuesta por textos de profundidad ensamblados mediante apoyo colaborativo entre periodistas de diversos países. Verdad Abierta recurre a diversos géneros, pero sobretodo crea informes especiales y reportajes, piezas expositivas e interpretativas con uso de diversas fuentes testimoniales y documentales. Por su parte Poderopedia crea con mayor énfasis fichas biográficas sobre personas, empresas y organizaciones. Cada ficha está conformada por sus conexiones, el perfil, el mapa de relaciones, documentos de intereses, fuentes de donde se obtiene la información, el río de noticias que circula en la red sobre el personaje, la empresa u organización en cuestión. Son todos contenidos cronoresistentes, con mayores tiempos de vigencia y permanencia en la red. 


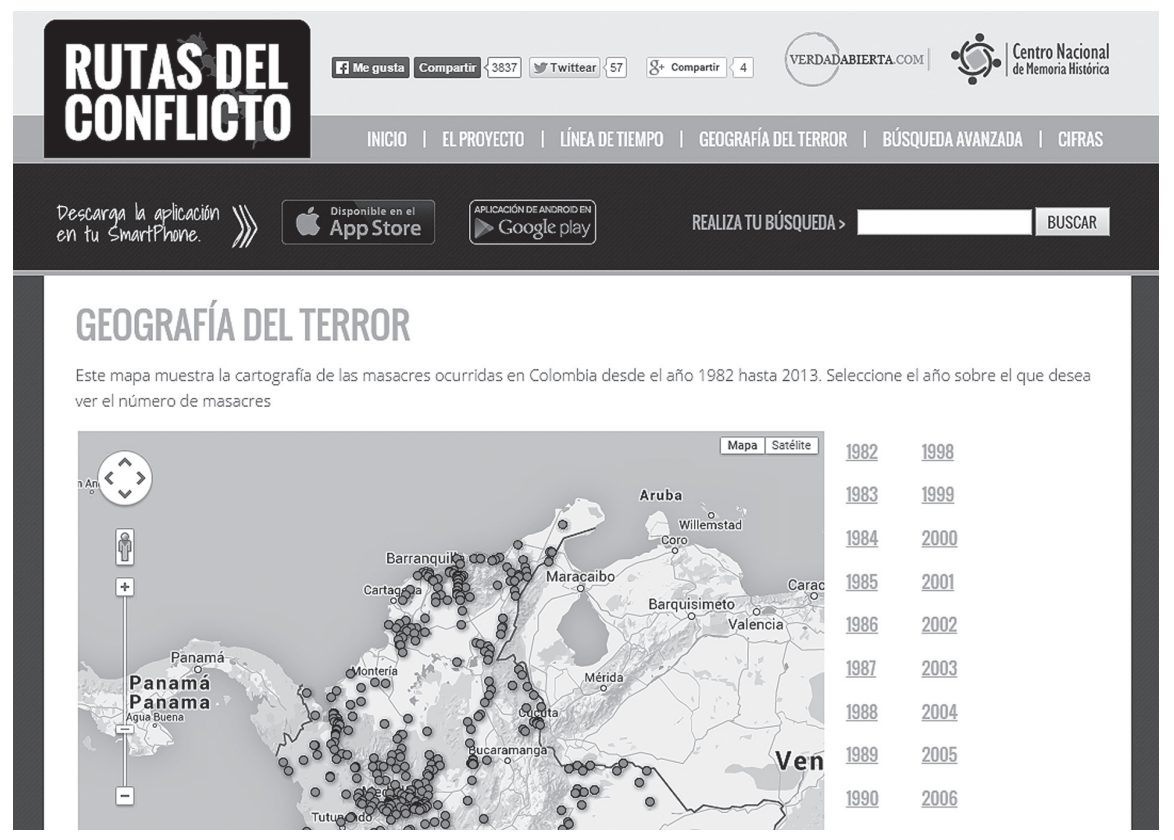

Imagen 5. Base de datos Rutas del Conflicto, realizada por el medio Verdad Abierta y el Centro de Memoria Histórica. http: / /rutasdelconflicto.com/

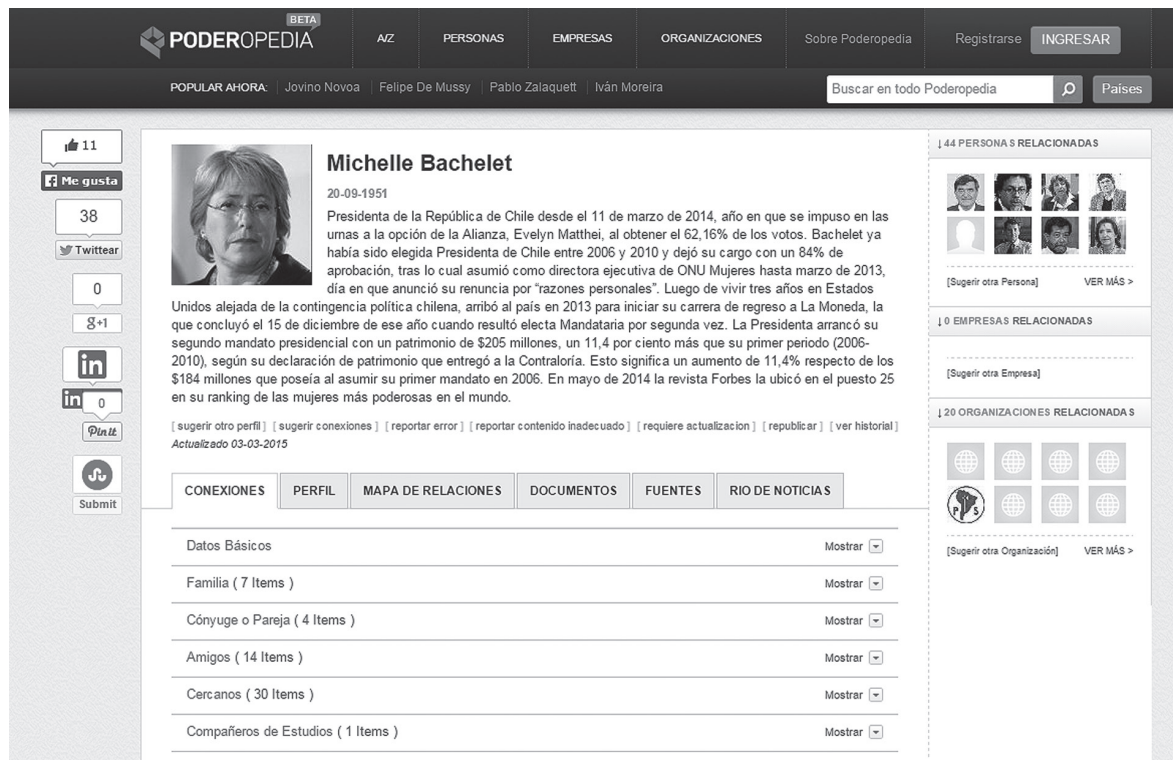

Imagen 6. zona biográfica de la presidenta chilena Michelle Bachelet en el medio Poderopedia. http://www.poderopedia.org/

\section{Conclusiones}

En 1999, ocho años después de iniciado el proceso de masificación de internet, Concha Edo investigaba por el impacto de internet en la noticia, las fuentes y los géneros. Señalaba que si bien hasta el momento los estudios sobre el discurso tradicional en la noticia han partido desde las características básicas de la actualidad, la novedad, la veracidad, la periodicidad y el interés público, los esquemas se han ido ampliando de forma creciente. 
Para 1998 Google era apenas un emprendimiento empresarial (startup) y en el año 2000 aún no se insinuaban fenómenos emergentes que cambiarían el panorama cultural global: Wikipedia solo fue lanzada en 2001, Facebook en 2004, Youtube en 2005, Twitter en 2006, el primer iphone fue lanzado en 2007, la primera tienda de aplicaciones de Apple en 2008 y las tabletas en 2010. Diversos emprendimientos en apenas una década generaron fenomenales mutaciones en vastos sectores de la cultura y sus apariciones develan el cada vez mayor carácter provisorio de nuestras investigaciones.

¿Podrán subsistir estos medios emergentes desvinculados de una actividad comercial fuerte?, ¿están asentando con sus prácticas un nuevo periodismo de la información o son apenas los albores y experimentos iniciales de un ecosistema aún en crecimiento? Si bien en la historia de la cultura no existen rupturas súbitas, en medio de la mutación tecnocultural, los medios emergentes seleccionados han desarrollado unos principios organizadores en sus prácticas que empiezan a insinuarse como patrón: la innovación de formatos de expresión, la apertura de sus data, la visibilización de las fuentes de financiación, el establecimiento de redes activas con otros medios, la construcción de una audiencia participativa en la producción de los contenidos, su concepción provisional de metamedios y el acceso transmediático a sus contenidos; movimientos tendientes hacia una paulatina reconceptualización del campo y del oficio. Un horizonte amplio que requiere nuestra atención.

\section{Notas}

\footnotetext{
${ }^{1}$ El informe detallado se puede consultar acá: http://www.naa.org/Trends-and-Numbers/CirculationVolume/Newspaper-Circulation-Volume.aspx

${ }^{2}$ http: / /www.statista.com/statistics/184682/us-daily-newspapers-by-circulation/

${ }^{3}$ http:/ / www.statista.com/statistics/377790/digital-newspaper-audience-by-number-of-visitors /

${ }^{4}$ http: / / ciperchile.cl/

${ }^{5}$ http://www.verdadabierta.com/

${ }^{6}$ http: / / lasillavacia.com/

${ }^{7}$ http: / / chequeado.com/

${ }^{8}$ http: / / connectas.org/

${ }^{9}$ http: / /www.poderopedia.org/co/

${ }^{10}$ Otros medios emergentes nativos que no hacen parte del corpus seleccionado sí lo hacen como el portal Kienyke (http:/ / www.kienyke.com), el cual nació como producto de la asociación entre un inversor privado y María Elvira Bonilla, una periodista de alta trayectoria.

${ }^{11}$ http: / / www.chicaspoderosas.org/

${ }^{12} \mathrm{http}: / /$ chequeado.com/chequeador\#/home

${ }^{13}$ Acá se puede leer la historia http:/ / lasillavacia.com/historia/15704
}

\section{Referencias}

Briggs, A. \& Burke, P., (2002). De Gutenberg a internet: Una historia social de los medios de comunicación. Madrid: Taurus.

Capra, F. (1998). La trama de la vida: Una nueva perspectiva de los sistemas vivos. Barcelona: Anagrama. Carr, N. (2011). Superficiales: ¿qué está haciendo internet con nuestras mentes? México, D.F: Taurus.Edo, C. (1999). Periodismo informativo e interpretativo: El impacto de Internet en la noticia, las fuentes y los géneros. Sevilla: Comunicación Social Ediciones y Publicaciones. García, K. (2011). Wikileaks y las expresiones del periodismo transformado. En Revista Nexus 9, 244-259. 
González, J. (2004). Repensar el periodismo:Transformaciones y emergencias del periodismo actual. Cali: Programa Editorial, Universidad del Valle.

Johnson, S. (2003). Sistemas emergentes: O qué tienen en común hormigas, neuronas, ciudades y software. Madrid: Turner.

Kozak, C., \& Castromán, E. (2012). Tecnopoéticas argentinas: Archivo blando de arte y tecnología. Buenos Aires: Caja Negra.

Landow, G. P. (2009). Hipertexto 3.0: La teoría crítica y los nuevos medios en una época de globalización. Barcelona: Paidós.

León, J. (Spring-summer 2011). La Silla Vacía. Eight Ways the Internet Changes the Way We Think about the News. En ReVista. Harvard Review of Latin America. Recuperado en mayo 20 de http:// revista.drclas.harvard.edu/book/la-silla-vac\%C3\%ADa

Levy, Pierre. (1999). Cibercultura. Sao Paulo: Editora 34.

Manovich, L. (2005). El lenguaje de los nuevos medios de comunicación: La imagen en la era digital. Barcelona: Paidós.

Manovich, L. (2013). El software toma el mando. Barcelona: Editorial UOC.

Martin, S. M. (2007). Teoría de la comunicación: La comunicación, la vida y la sociedad. Madrid: McGraw Hill.

Martín-Barbero, J. (2012). Mutación tecnocultural, convergencia entre tecnología y cultura. Material de trabajo en el Seminario La Comunicación Universidad / Sociedad en la mutación de las tecnicidades, del conocimiento y del arte. Cali: Universidad del Valle.

Martín-Barbero, J. (1987). De los medios a las mediaciones: Comunicación, cultura y hegemonía. México: Ediciones G. Gili.

Mattelart, A. (2002). Historia de la sociedad de la información. Barcelona: Paidóss.

McLuhan, M. (1996). Comprender los medios de comunicación: Las extensiones del ser humano. Barcelona: Paidós.

Morin, E. (1994). Introducción al pensamiento complejo. Barcelona: Gedisa.

Scolari, C. (2008). Hipermediaciones. Elementos para una Teoría de la Comunicación Digital Interactiva. Barcelona: Gedisa.

Scolari, C. A. (2013). Narrativas Transmedia: Cuando todos los medios cuentan. Barcelona: Centro Libros PAPF, S.L.U.

Chiappe, Doménico (2011). WikiLeaks y el mal periodismo. En Frontera D. Recuperado en junio 6 de http: / / www.fronterad.com/?q=wikileaks-y-el-mal-periodismo\&page $=0,0$

Domscheit-Berg, Daniel. (2011). Dentro deWikiLeaks. Mi etapa en la web más peligrosa del mundo. Barcelona: Roca editorial.

Eco, Umberto (febrero 12 de 2010). Hackers vengeurs et espions en diligence. En Libération.fr. Recuperado en mayo 15 de http://www.liberation.fr/monde/01012305696-hackers-vengeurset-espions-en-diligence

León, Juanita (spring-summer 2011). La Silla Vacía. Eight Ways the Internet Changes the Way We Think about the News. En ReVista. Harvard Review of Latin America. Recuperado en mayo 20 de http: / / www.drclas.harvard.edu/publications/revistaonline/spring-summer-2011 / la-silla-vacía

Ramonet, Ignacio (2002). Los periodistas están en vías de extinción. En Sala de prensa. Edición 46. Año IV, Vol. 2 Recuperado de http://www.saladeprensa.org/art382.htm, en mayo 15 de 2011.

\section{Sitios Web}

Ciper, Centro de investigación periodística. http: / / ciperchile.cl/

Verdad Abierta. Conflicto armado en Colombia. http: / / www.verdadabierta.com/

La silla vacía. http:/ /lasillavacia.com/

Chequeado La verificación del discurso público. http://chequeado.com/

Connectas, plataforma de periodismo para las Américas. http: / / connectas.org

Poderopedia. Quién es quién en los negocios y en la política. http://www.poderopedia.org/

Recibido: abril 30 / Aprobado: junio 1 de 2015 